\title{
Active vibration control of a nonlinear beam with self- and external excitations
}

\author{
J. Warminski ${ }^{\mathrm{a}, *}$, M.P. Cartmell ${ }^{\mathrm{b}}$, A. Mitura ${ }^{\mathrm{a}}$ and M. Bochenski ${ }^{\mathrm{a}}$

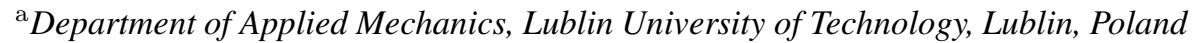 \\ ${ }^{\mathrm{b}}$ Department of Mechanical Engineering, University of Sheffield, Sheffield, UK
}

\begin{abstract}
An application of the nonlinear saturation control (NSC) algorithm for a self-excited strongly nonlinear beam structure driven by an external force is presented in the paper. The mathematical model accounts for an Euler-Bernoulli beam with nonlinear curvature, reduced to first mode oscillations. It is assumed that the beam vibrates in the presence of a harmonic excitation close to the first natural frequency of the beam, and additionally the beam is self-excited by fluid flow, which is modelled by a nonlinear Rayleigh term for self-excitation. The self- and externally excited vibrations have been reduced by the application of an active, saturation-based controller. The approximate analytical solutions for a full structure have been found by the multiple time scales method, up to the first-order approximation. The analytical solutions have been compared with numerical results obtained from direct integration of the ordinary differential equations of motion. Finally, the influence of a negative damping term and the controller's parameters for effective vibrations suppression are presented.
\end{abstract}

Keywords: Active control, saturation, self-excited vibrations, external harmonic force, interactions, nonlinear beam oscillations, multiple scales method

\section{Introduction}

Applications of controllers using the saturation phenomenon to reduce a system's vibrations have been intensively developed in recent years. At first the advantage of this phenomenon was clear for mechanical structures. Golnaraghi [6] proposed a system, where a sliding mass-spring-dashpot mechanism, placed at the free end of a cantilever beam, was used to reduce the free vibrations of such a beam. Nonlinear quadratic coupling between the beam and the slider, and also 2:1 internal resonance (with the frequency of the slider being twice the fundamental beam frequency) led to energy transfer between the beam and the slider. The author investigated this system with and without damping. The inclusion of damping caused an absorption of the beam's vibrational energy, dissipated by the slider. A similar approach was demonstrated by Duquette et al. [3,4]. However, in that case the controller's role was played by a DC motor with a pendulum attached to the motor shaft. This system was mounted at the free end of a flexible cantilever beam. The authors presented their results of analytical and numerical investigations (in part 1) and experimental tests were subsequently shown (in part 2). Harmonic, subharmonic and superharmonic responses of the beam were observed for selected parameters. Oueini et al. [13] presented a controller based on the saturation phenomena, operated as a vibration absorber for a linear model of a cantilever beam. A piezoelectric element was used as an actuator and a strain gauge as a sensor. By using the multiple time scales method the influences of loop gains and damping of the controller were both studied. Additionally sensitivity to the controller's initial conditions and the saturation phenomena was examined. Jun et al. [7] applied a saturation controller to a nonlinear plant using standard PZT patches. Based on analytical solutions these authors showed that the nonlinear absorber (NSC) was

\footnotetext{
* Corresponding author: Jerzy Warminski, Department of Applied Mechanics, Lublin University of Technology, Nadbystrzycka 36, 20-618 Lublin, Poland. Fax: +48 081538 4205; E-mail: j.warminski@ pollub.pl.
} 
globally stable, as distinct from a linear model where increasing the feedback gain value might instead lead to instability. However, for a nonlinear absorber the power requirement was found to be greater than that for the linear system. Xu et al. [25] investigated the effect of a time delay in a structure consisting of a linear beam and the NSC absorber. The analytical results based on the multiple scales method revealed much more complex dynamics for the system. The presence of the time delay widened or reduced the frequency bandwidth of effective vibration reduction. Warminski et al. [22] analysed the influence of the controller parameters, and also the hard or soft geometrical plant nonlinearities, to the dynamical response of the system undergoing NSC. Moreover, the effectiveness of the selected control algorithms to vibration suppression of a strongly nonlinear beam structure was compared and discussed. The results were obtained by means of analytical, numerical and experimental tests. Macro fibre composite (MFC) actuators were implemented for the real system and these allowed the required vibration level to be activated.

An application for a controller based on the saturation phenomenon for self-excited systems was presented by ElBadawy and Nasr El-Deen [5]. They demonstrated possible vibration suppression of a self-excited system, described by a van der Pol model. The analytical solutions exhibited a significant influence coming from the controller's damping, and confirmed that for a perfectly tuned system (where the frequency of the controller was half of the fundamental plant's frequency) the response of the system was independent of the original system parameters. Jun et al. [8] took into account the same van der Pol oscillator with NSC, but additionally they investigated the influence of feedback gains. Moreover, both perturbation and direct numerical integration solutions were presented.

The saturation based control method gives good results if the dynamics of the plant are known and the controller is properly tuned (Cartmell [2], Nayfeh [10]). However, in many examples two (or more) different vibration types exist simultaneously, and then additional interactions may occur. Systems with self- and parametric or external excitations have been intensively studied by Tondl and Ecker [19], Tondl and Nabergoj [20] or Pust and Tondl [15]. They have found that interactions between self- and periodically excited systems lead to a quasi-periodic response, but in selected frequency domains the frequency quenching phenomenon is seen to take place. This phenomenon, sometimes called frequency locking, occurs after an inverse Neimark-Sacker bifurcation. The quenching phenomenon of selfexcited systems which have important practical meanings in fluid-structure interactions, was extensively studied by Abadi [1] and Verhulst [21]. In some examples a self-exited system may be simultaneously excited parametrically and externally. Then, the frequency locking zones may change radically for some combinations of parameters. It has been shown by Szabelski and Warminski [16-18] that a small external force may change the system's response. In such a case an internal loop occurs in the amplitude-frequency response curve. This phenomenon leads to five possible steady states in the frequency locking zones. Moreover, apart from the regular dynamics, interactions between self- parametric or external excitations may lead to chaotic or hyperchaotic dynamics (Warminski [23,24]).

This paper concentrates on an application of a nonlinear saturation controller to a system where two kinds of excitation appear simultaneously. One of them is harmonic excitation caused by support motion of the cantilever beam, the second comes from nonlinear damping with a negative linear part, and this models self-excitation. Additional geometrical and inertial nonlinearities of the beam are also taken into account. The dynamics of this controlled nonlinear structure are compared with those of a nonlinear plant model with classical linear and positive damping. To the authors' knowledge there have been no studies presented in the literature of this kind of system. The first-order approximate analytical solutions thus obtained are compared with the results of direct numerical integration.

\section{Model of the structure}

The model of the beam structure, together with characteristic parameters, is presented in Fig. 1 . The beam is made of a glass-epoxy composite material with a chosen fibre orientation of 0/90/45/-45/45/90/0 and physical parameters taking the following values: Young's modulus $E=0.255 \times 10^{5} \mathrm{MPa}$, mass density $\rho=2100 \mathrm{~kg} / \mathrm{m}^{3}$, width $b=$ $12.8 \mathrm{~mm}$, height $h=1 \mathrm{~mm}$ and length of the beam $L=236 \mathrm{~mm}$. At the end of the composite beam a lumped mass $M$ is attached. By varying this mass one may change the characteristics of the system. The cantilever beam is mounted on an armature of an electrodynamic shaker, this being a source of excitation along the $X$ axis. The kinematic excitation is written as

$$
x=x_{0} \sin (\Omega t)
$$




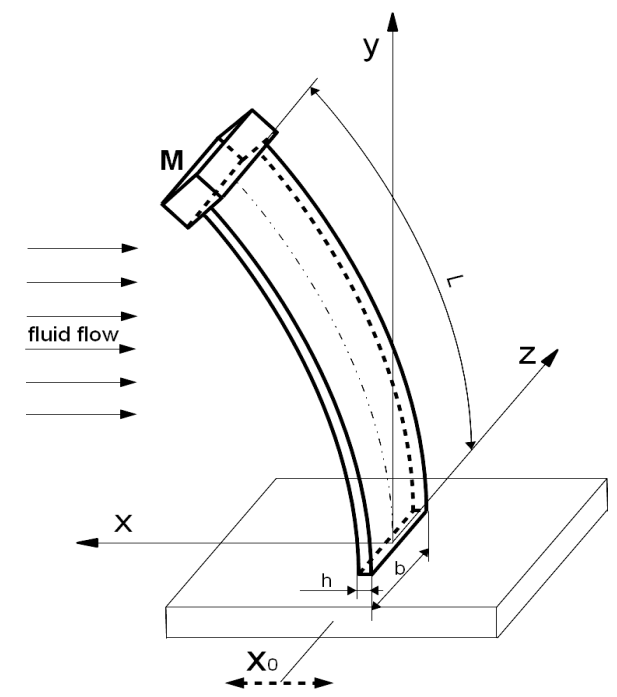

Fig. 1. Model of the nonlinear beam with self- and external excitations.

The nonlinear mathematical model which can be used for large beam deflection analysis has been adopted from the literature (Nayfeh [10], Jun et al. [8], El-Badawy and Nasr El-Deen [5]). Considering only the first bending mode, the differential equation of the beam (the plant) is written in the dimensionless form:

$$
\ddot{x}_{1}+\left(-\alpha_{1} \dot{x}_{1}+\beta_{1} \dot{x}_{1}^{3}\right)+\omega_{01}^{2} x_{1}+\gamma_{1} x_{1}^{3}+\delta\left(x_{1} \dot{x}_{1}^{2}+x_{1}^{2} \ddot{x}_{1}\right)=x_{0} \mu \Omega^{2} \sin (\Omega t)+U
$$

where $x_{1}$ is the generalized coordinate. Nonlinear damping (self-excitation) is represented by Rayleigh's function, where $\alpha_{1}$ is the negative viscous damping coefficient and $\beta_{1}$ is the cubic damping coefficient. The nonlinear damping term is considered as a phenomenological model of self-excited vibrations. The dimensionless linear natural frequency $\omega_{01}$ is a ratio of the natural frequency of the composite beam with the lumped mass with respect to that of the reference beam without the lumped mass. This means that $\omega_{01}=1$ if $M=0$. Coefficients $\gamma_{1}, \delta$ describe the geometrical and inertia nonlinearities, respectively. The kinematic support motion $x_{0} \sin (\Omega t)$ produces external excitation $x_{0} \mu \Omega^{2} \sin (\Omega t)$ where $\mathrm{x}_{0}$ and $\Omega$ are the amplitude and frequency respectively of the support motion, and $\mu$ is a constant.

On the right hand side of the differential Eq. (2) is an added control force $U$. In this paper the nonlinear saturation control (NSC) method is investigated, therefore the control law is given by

$$
U=g_{1} x_{2}^{2}
$$

where $g_{1}$ is a positive control feedback gain. The input signal in the quadratic term $x_{2}^{2}$ is defined by (Nayfeh [10])

$$
\ddot{x}_{2}+\alpha_{2} \dot{x}_{2}+\omega_{02}^{2} x_{2}=g_{2} x_{2} x_{1}
$$

In the NSC controller of Eq. (4) the parameters $\omega_{02}, \alpha_{2}$ are the controller's natural frequency and damping coefficient, respectively. The parameter $g_{2}$ is the control gain. According to the saturation control strategy (Oueini et al. [13], Nayfeh [10]) the controller is tuned to the plant frequency such that: $\omega_{02}=\frac{1}{2} \omega_{01}$.

\section{Perturbation analysis}

Approximate analytical solutions are sought by means of the multiple time scales method (Nayfeh [11]). Assuming that the system is weakly nonlinear and then introducing a formal small parameter $\varepsilon$, the set of Eqs (2)-(4) is transformed to the form

$$
\left\{\begin{array}{l}
\ddot{x}_{1}+\omega_{01}^{2} x_{1}=\varepsilon\left[\tilde{\alpha}_{1} \dot{x}_{1}-\tilde{\beta}_{1} \dot{x}_{1}^{3}-\tilde{\gamma}_{1} x_{1}^{3}-\tilde{\delta}\left(x_{1} \dot{x}_{1}^{2}+x_{1}^{2} \ddot{x}_{1}\right)+\tilde{x}_{0} \mu \Omega^{2} \sin (\Omega t)+\tilde{g}_{1} x_{2}^{2}\right] \\
\ddot{x}_{2}+\omega_{02}^{2} x_{2}=\varepsilon\left(-\tilde{\alpha}_{2} \dot{x}_{2}+\tilde{g}_{2} x_{2} x_{1}\right)
\end{array}\right.
$$


where the dimensionless parameters of the system are assumed to be ordered by the formal small parameter $\varepsilon$ as follows

$$
\alpha_{1}=\varepsilon \tilde{\alpha}_{1}, \quad \beta_{1}=\varepsilon \tilde{\beta}_{1}, \quad \gamma_{1}=\varepsilon \tilde{\gamma}_{1}, \quad \delta_{1}=\varepsilon \tilde{\delta}_{1}, \quad x_{0}=\varepsilon \tilde{x}_{0}, \quad g_{1}=\varepsilon \tilde{g}_{1}, \quad \alpha_{2}=\varepsilon \tilde{\alpha}_{2}, \quad g_{2}=\varepsilon \tilde{g}_{2}
$$

The above ordering takes into account the fact that plant's response is relatively small, thus the nonlinear terms $\tilde{\gamma}_{1} x_{1}^{3}$ and $\tilde{\delta}\left(x_{1} \dot{x}_{1}^{2}+x_{1}^{2} \ddot{x}_{1}\right)$ are small even if values of $\gamma_{1}$ and $\delta$ are relatively high.

For simplicity the tilde is hereafter neglected. The solution for the coordinates $x_{1}$ and $x_{2}$ is assumed in the usual form of a series in the small parameter $\varepsilon$

$$
\begin{aligned}
& x_{1}(t, \varepsilon)=x_{10}\left(T_{0}, T_{1}\right)+\varepsilon x_{11}\left(T_{0}, T_{1}\right)+\ldots \\
& x_{2}(t, \varepsilon)=x_{20}\left(T_{0}, T_{1}\right)+\varepsilon x_{21}\left(T_{0}, T_{1}\right)+\ldots
\end{aligned}
$$

Coordinates $x_{j 0}\left(T_{0}, T_{1}\right), x_{j 1}\left(T_{0}, T_{1}\right),(j=1,2)$ are, respectively, the zeroth and first order perturbations. Dimensionless time is also expressed by a series of the small parameter,

$$
t=T_{0}+\varepsilon T_{1} \ldots
$$

where $T_{0}, T_{1}$ are respectively the fast and slow time scales. Such time scale definitions result in the following formulae for the first and the second time derivatives

$$
\begin{aligned}
\frac{\mathrm{d}}{\mathrm{d} t} & =\frac{\partial}{\partial T_{0}}+\varepsilon \frac{\partial}{\partial T_{1}}=D_{0}+\varepsilon D_{1} \ldots \\
\frac{\mathrm{d}^{2}}{\mathrm{~d} t^{2}} & =D_{0}^{2}+2 \varepsilon D_{0} D_{1}+\ldots
\end{aligned}
$$

where $D_{n}^{m}=\frac{\partial^{m}}{\partial T_{n}}$ means the $m^{\text {th }}$ order partial derivative with respect to the $n^{\text {th }}$ time-scale.

We seek solutions near the fundamental natural frequency of the beam taking into account the controller which is tuned for internal resonance $\omega_{02}=\omega_{01} / 2$. The frequency of excitation $\Omega$ is close to the first natural frequency of the beam and is twice the natural frequency of the controller (close to the principal parametric resonance). Under such conditions the response of the beam is harmonic, while the response of the controller is subharmonic.

Assuming that $\Omega \approx \omega_{01}$, we can write

$$
\omega_{01}^{2}=\Omega^{2}-\varepsilon \sigma_{1}
$$

where $\sigma_{1}$ is the frequency detuning parameter.

Substituting solutions Eq. (6) into Eq. (5), taking into account the derivative definitions Eqs (8) and (9), and expressing the natural frequency according to Eq. (10), then after grouping terms with respect to order $\varepsilon$ we get a set of differential equations in the successive perturbation orders.

$\varepsilon^{0}-$ order

$$
\begin{aligned}
& D_{0}^{2} x_{10}+\Omega^{2} x_{10}=0 \\
& D_{0}^{2} x_{20}+\frac{1}{4} \Omega^{2} x_{20}=0
\end{aligned}
$$

$\varepsilon^{1}-$ order

$$
\begin{aligned}
D_{0}^{2} x_{11}+\Omega^{2} x_{11}= & \sigma_{1} x_{10}-2 D_{0} D_{1} x_{10}+g_{1} x_{20}^{2}+\alpha_{1} D_{0} x_{10}-\beta_{1}\left(D_{0} x_{10}\right)^{3} \\
& -\delta x_{10}\left[\left(D_{0} x_{10}\right)^{2}+x_{10} D_{0}^{2} x_{10}\right]-\gamma x_{10}^{3}+x_{0} \mu \Omega^{2} \sin \Omega T_{0} \\
D_{0}^{2} x_{21}+\frac{1}{4} \Omega^{2} x_{21}= & \frac{1}{4} \sigma_{1} x_{20}-2 D_{0} D_{1} x_{20}-\alpha_{2} D_{0} x_{20}+g_{2} x_{10} x_{20}
\end{aligned}
$$


The solutions of Eq. (11) have the conventional complex form

$$
\begin{aligned}
& x_{10}\left(T_{0}, T_{1}, T_{2}\right)=A_{1}\left(T_{1}, T_{2}\right) \exp \left(i \Omega T_{0}\right)+\bar{A}_{1}\left(T_{1}, T_{2}\right) \exp \left(-i \Omega T_{0}\right) \\
& x_{20}\left(T_{0}, T_{1}, T_{2}\right)=A_{2}\left(T_{1}, T_{2}\right) \exp \left(i \Omega T_{0}\right)+\bar{A}_{2}\left(T_{1}, T_{2}\right) \exp \left(-i \Omega T_{0}\right)
\end{aligned}
$$

where $i=\sqrt{-1}$ is the imaginary unit, $A_{1}, A_{2}$ are the complex amplitudes of the plant and the controller respectively and $\bar{A}_{1}, \bar{A}_{2}$ their complex conjugates. Solutions Eq. (13) are substituted into Eq. (12) and, after grouping the terms in their proper exponential functions, we get

$$
\begin{aligned}
D_{0}^{2} x_{11}+\Omega^{2} x_{11} & =2 g_{1} A_{2} \bar{A}_{2}-\left(\gamma_{1}-2 \delta \Omega^{2}-i \beta_{1} \Omega\right) A_{1}^{3} e^{i 3 \Omega T_{0}}+S T_{11} e^{i \Omega T_{0}}+c c \\
D_{0}^{2} x_{21}+\frac{1}{4} \Omega^{2} x_{21} & =\frac{3}{2} g_{2} A_{1} A_{2}+S T_{21} e^{i \frac{1}{2} \Omega T_{0}}+c c
\end{aligned}
$$

where $c c$ means complex conjugate functions. $S T_{11}, S T_{21}$ are those terms which will generate secular terms in the solution of Eq. (14). Therefore, to avoid this situation the $S T_{11}, S T_{21}$ have to be set to zero, thus we have

$$
\begin{aligned}
& -\sigma_{1} A_{2}-g_{1} A_{2}^{2}+\frac{1}{2} i x_{0} \mu \Omega^{2}-i \alpha_{1} \Omega A_{1}+\left(3 i \beta \Omega^{3}+3 \gamma_{1}-2 \delta \Omega^{2}\right) A_{1}^{2} \bar{A}_{1}+2 i \Omega D_{1} A_{1}=0 \\
& -\frac{1}{4} \sigma_{1} A_{2}-g_{2} A_{1} \bar{A}_{2}+\frac{1}{2} i \alpha_{2} \Omega A_{2}+i \Omega D_{1} A_{2}=0
\end{aligned}
$$

Next by rejecting the $S T_{11}, S T_{21}$ terms we may determine the particular solutions of Eq. (14)

$$
\begin{aligned}
& x_{11}=\frac{2}{\Omega^{2}} g_{1} A_{2} \bar{A}_{2}+\frac{1}{8 \Omega^{2}}\left(-i \beta_{1} \Omega^{3}+\gamma_{1}-2 \delta \Omega^{2}\right) A_{1}^{3} e^{i 3 \Omega T 0}+c c \\
& x_{21}=-\frac{1}{2 \Omega^{2}} g_{2} A_{1} A_{2} e^{i \frac{3}{2} \Omega T 0}+c c
\end{aligned}
$$

Then we may formulate the modulation equations for the complex amplitudes $A_{1}, A_{2}$. The amplitude derivatives with respect to time take the forms

$$
\begin{aligned}
& \frac{d A_{1}}{d t}=\varepsilon D_{1} A_{1} \\
& \frac{d A_{2}}{d t}=\varepsilon D_{1} A_{2}
\end{aligned}
$$

Derivatives $D_{1} A_{1}$ and $D_{1} A_{2}$ are found from Eq. (15), and then using Eq. (17) we get

$$
\begin{aligned}
\frac{d A_{1}}{d t} & =\varepsilon \frac{1}{2 \Omega}\left\{-\frac{1}{2} x_{0} \mu \Omega^{2}+\left(\alpha_{1} \Omega-i \sigma_{1}\right) A_{1}-i g_{1} A_{2}^{2}+\left(3 i \gamma_{1}-2 i \delta \Omega^{2}-3 \beta_{1} \Omega^{3}\right) A_{1}^{2} \bar{A}_{1}\right\} \\
\frac{d A_{2}}{d t} & =\varepsilon\left\{-\frac{A_{2}}{4 \Omega}\left(2 \alpha_{2} \Omega+i \sigma_{1}\right)-\frac{i g_{2} A_{1} \bar{A}_{2}}{\Omega}\right\}
\end{aligned}
$$

Expressing the complex amplitudes $A_{1}, A_{2}$ in the polar form

$$
A_{1}=\frac{1}{2} a_{1} e^{i \phi_{1}} \quad A_{2}=\frac{1}{2} a_{2} e^{i \phi_{2}}
$$

and then separating the real and imaginary parts of Eq. (18), we get the modulation equations for amplitudes $a_{1}, a_{2}$ and phases $\phi_{1}, \phi_{2}$.

The modulation equations, which represent the so-called slow flow have the form

$$
\begin{aligned}
\frac{d a_{1}}{d t} & =\varepsilon\left(-\frac{1}{2} x_{0} \mu \Omega \cos \phi_{1}+\frac{1}{2} \alpha_{1} a_{1}-\frac{3}{8} \beta_{1} \Omega^{2} a_{1}^{3}-\frac{1}{4 \Omega} g_{1} a_{2}^{2} \sin \left(\phi_{1}-2 \phi_{2}\right)\right) \\
\frac{d \phi_{1}}{d t} & =\varepsilon\left(-\frac{\sigma_{1}}{2 \Omega}+\frac{3 \gamma_{1} a_{1}^{2}}{8 \Omega}-\frac{1}{4} \delta \Omega a_{1}^{2}-\frac{g_{1} a_{2}^{2}}{4 \Omega a_{1}} \cos \left(\phi_{1}-2 \phi_{2}\right)+\frac{x_{0} \mu \Omega}{2 a_{1}} \sin \phi_{1}\right) \\
\frac{d a_{2}}{d t} & =\varepsilon\left(-\frac{1}{2} \alpha_{2} a_{2}+\frac{1}{2 \Omega} g_{2} a_{1} a_{2} \sin \left(\phi_{1}-2 \phi_{2}\right)\right)
\end{aligned}
$$




$$
\frac{d \phi_{2}}{d t}=\varepsilon\left(-\frac{\sigma_{1}}{4 \Omega}-\frac{g_{2} a_{1}}{2 \Omega} \cos \left(\phi_{1}-2 \phi_{2}\right)\right)
$$

By taking into account the particular solutions Eqs (13) and (16), and the complex amplitudes in the polar form Eq. (19), then according to Eq. (6) we obtain approximate solutions to first order approximation

$$
\begin{aligned}
x_{1}= & a_{1} \cos \left(\Omega T_{0}+\phi_{1}\right)+\varepsilon \frac{1}{2 \Omega^{2}}\left[g_{1} a_{2}^{2}+\frac{1}{8}\left(-\delta \Omega^{2}+\frac{1}{2} \gamma_{1}\right) a_{1}^{3} \cos 3\left(\Omega T_{0}+\phi_{1}\right)\right. \\
& \left.+\frac{1}{16} \beta_{1} \Omega^{3} a_{1}^{3} \sin 3\left(\Omega T_{0}+\phi_{1}\right)\right] \\
x_{2}= & a_{2} \cos \left(\frac{1}{2} \Omega T_{0}+\phi_{2}\right)+\varepsilon \frac{1}{4 \Omega^{2}} g_{2} a_{1} a_{2} \cos \left(\frac{3}{2} \Omega T_{0}+\phi_{1}+\phi_{2}\right)
\end{aligned}
$$

Amplitudes $a_{1}, a_{2}$ and phases $\phi_{1}, \phi_{2}$ have to be found from the modulation Eq. (20), or in the steady state from the equivalent algebraic equations.

\section{Stability analysis}

The stability of the periodic solutions is analysed by using the approximate slow-flow first order differential Eq. (20), which can be written in shortened form as follows

$$
\begin{aligned}
\frac{d a_{1}}{d t} & =F_{1}\left(a_{1}, \phi_{1}, a_{2}, \phi_{2}\right) \\
\frac{d \phi_{1}}{d t} & =F_{2}\left(a_{1}, \phi_{1}, a_{2}, \phi_{2}\right) \\
\frac{d a_{2}}{d t} & =F_{3}\left(a_{1}, \phi_{1}, a_{2}, \phi_{2}\right) \\
\frac{d \phi_{2}}{d t} & =F_{4}\left(a_{1}, \phi_{1}, a_{2}, \phi_{2}\right)
\end{aligned}
$$

where the functions $F_{1}, F_{2}, F_{3}, F_{4}$ are the right hand sides of Eq. (20). In the steady state, Eq. (22) are equal to zero, therefore

$$
\begin{aligned}
& F_{1}\left(a_{1}, \phi_{1}, a_{2}, \phi_{2}\right)=0 \\
& F_{2}\left(a_{1}, \phi_{1}, a_{2}, \phi_{2}\right)=0 \\
& F_{3}\left(a_{1}, \phi_{1}, a_{2}, \phi_{2}\right)=0 \\
& F_{4}\left(a_{1}, \phi_{1}, a_{2}, \phi_{2}\right)=0
\end{aligned}
$$

By introducing perturbations $\delta a_{1}, \delta \phi_{1}, \delta a_{2}, \delta \phi_{2}$ to the fixed points, the variational differential equations have the form

$$
\begin{aligned}
& \frac{d \delta a_{1}}{d t}=\left(\frac{\delta F_{1}}{\delta a_{1}}\right) \delta a_{1}+\left(\frac{\delta F_{1}}{\delta \phi_{1}}\right) \delta \phi_{1}+\left(\frac{\delta F_{1}}{\delta a_{2}}\right) \delta a_{2}+\left(\frac{\delta F_{1}}{\delta \phi_{2}}\right) \delta \phi_{2} \\
& \frac{d \delta \phi_{1}}{d t}=\left(\frac{\delta F_{2}}{\delta a_{1}}\right) \delta a_{1}+\left(\frac{\delta F_{2}}{\delta \phi_{1}}\right) \delta \phi_{1}+\left(\frac{\delta F_{2}}{\delta a_{2}}\right) \delta a_{2}+\left(\frac{\delta F_{2}}{\delta \phi_{2}}\right) \delta \phi_{2} \\
& \frac{d \delta a_{2}}{d t}=\left(\frac{\delta F_{3}}{\delta a_{1}}\right) \delta a_{1}+\left(\frac{\delta F_{3}}{\delta \phi_{1}}\right) \delta \phi_{1}+\left(\frac{\delta F_{3}}{\delta a_{2}}\right) \delta a_{2}+\left(\frac{\delta F_{3}}{\delta \phi_{2}}\right) \delta \phi_{2} \\
& \frac{d \delta \phi_{2}}{d t}=\left(\frac{\delta F_{4}}{\delta a_{1}}\right) \delta a_{1}+\left(\frac{\delta F_{4}}{\delta \phi_{1}}\right) \delta \phi_{1}+\left(\frac{\delta F_{4}}{\delta a_{2}}\right) \delta a_{2}+\left(\frac{\delta F_{4}}{\delta \phi_{2}}\right) \delta \phi_{2}
\end{aligned}
$$




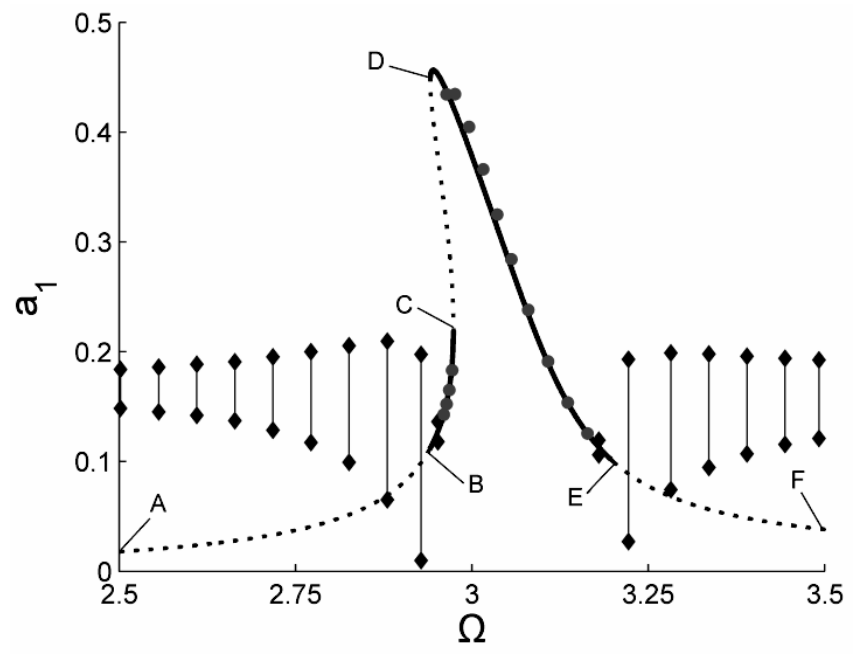

Fig. 2. Analytical and numerical results for the system without control. Analytical solution: solid line - stable solution, dotted line - unstable solution. Numerical simulation: circles - amplitude of periodic motion, diamonds - maximum and minimum amplitude of positive part of envelope of quasi-periodic motion; $x_{0}=0.01$.

with the characteristic determinant of Eq. (24) expressed as follows

$$
\left|\begin{array}{llll}
\frac{\delta F_{1}}{\delta a_{1}}-\lambda & \frac{\delta F_{1}}{\delta \phi_{1}} & \frac{\delta F_{1}}{\delta a_{2}} & \frac{\delta F_{1}}{\delta \phi_{2}} \\
\frac{\delta F_{2}}{\delta a_{1}} & \frac{\delta F_{2}}{\delta \phi_{1}}-\lambda & \frac{\delta F_{2}}{\delta a_{2}} & \frac{\delta F_{2}}{\delta \phi_{2}} \\
\frac{\delta F_{3}}{\delta a_{1}} & \frac{\delta F_{3}}{\delta \phi_{1}} & \frac{\delta F_{3}}{\delta a_{2}}-\lambda & \frac{\delta F_{3}}{\delta \phi_{2}} \\
\frac{\delta F_{4}}{\delta a_{1}} & \frac{\delta F_{4}}{\delta \phi_{1}} & \frac{\delta F_{4}}{\delta a_{2}} & \frac{\delta F_{4}}{\delta \phi_{2}}-\lambda
\end{array}\right|=0
$$

The stability of the approximate solutions Eq. (20) depends on the roots $\lambda$ of the characteristic Eq. (25). The solutions are obviously stable if the roots of the characteristic equation have negative real parts.

\section{Analytical and numerical results}

Numerical simulations and analytical investigation have been performed for a composite beam with the physical parameters given in Section 2. On this basis the dimensionless parameters of the system take these values: $\alpha_{1}=0.01$, $\beta_{1}=0.05, \omega_{01}=3.06309, \gamma_{1}=14.4108, \delta=3.2746, \mu=0.89663$. The amplitude and frequency of excitation are varied, respectively, in these ranges: $x_{0} \in(0,0.1), \Omega \in(2.4,3.6)$. The NSC controller parameters are chosen to be: $g_{1}=0.01, g_{2}=2, \omega_{02}=\omega_{01} / 2$ and the damping coefficient of the controller $\alpha_{2}$ is varied from 0 to 0.6 .

\subsection{Nonlinear system without control}

In order to design a proper control strategy we firstly investigate the dynamics of the nonlinear beam without control. The resonance curve for a steady state has been determined from Eq. (20) by setting them to zero. The stability of the approximate analytical solutions has been determined by calculating the roots of the characteristic Eq. (25). Then the analytical results are compared with those of direct numerical integration of Eq. (5). In Fig. 2 the analytical results are denoted by solid or dotted lines which correspond to stable or unstable solutions, respectively. The resonance curve is divided into five regions, two of which are stable and three are unstable. The first part of 


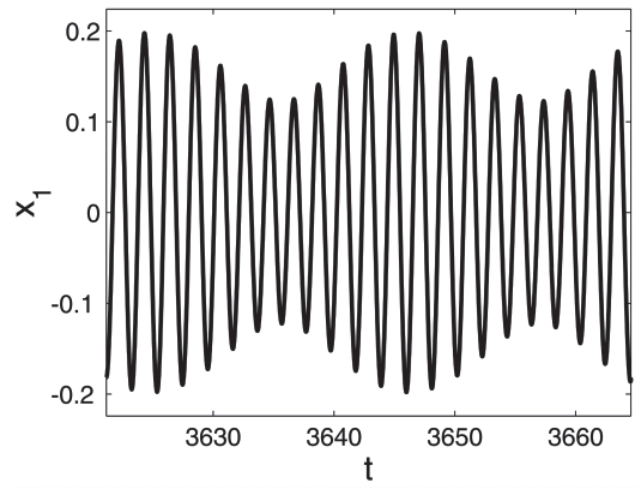

(a)

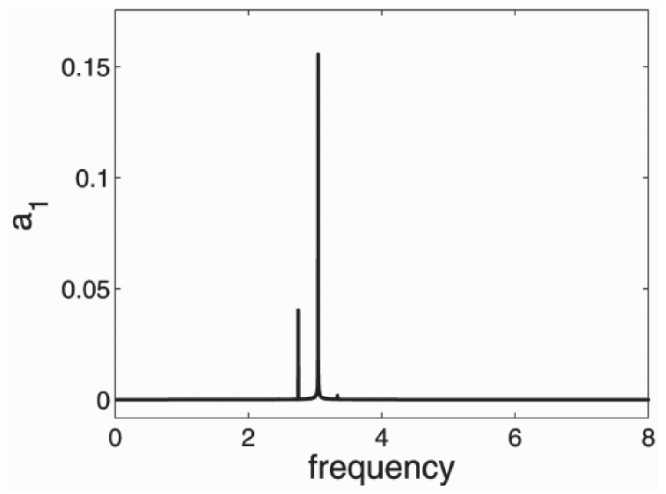

(b)

Fig. 3. Time series (a) and fast Fourier transform (b) for quasi-periodic beam motion; $x_{0}=0.01, \Omega=2.75$.

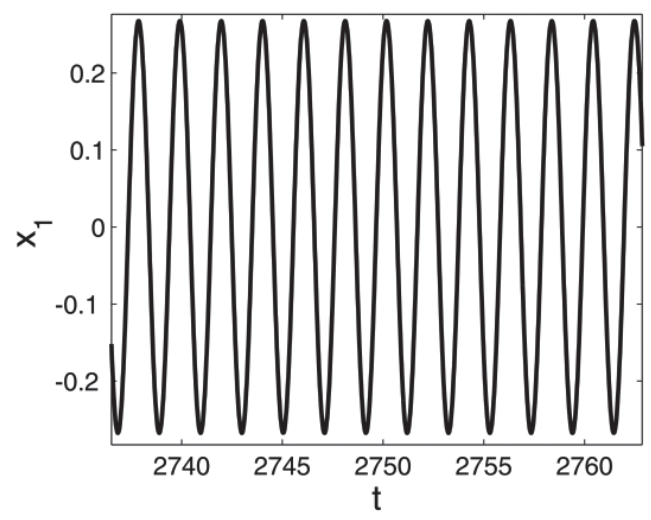

(a)

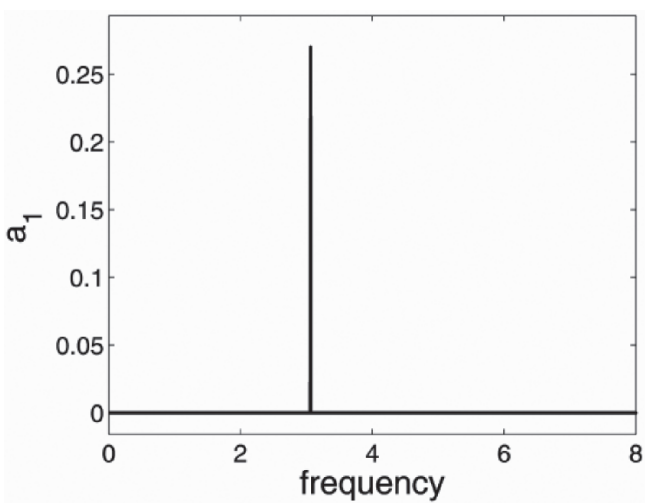

(b)

Fig. 4. Time series (a) and fast Fourier transform (b) for periodic beam response; $x_{0}=0.01, \Omega=3.06309$.

the analytical curve, from point $\mathrm{A}$ to $\mathrm{B}$, is unstable, with the roots of the characteristic equation being conjugate complex numbers with positive real parts. This solution corresponds to an unstable focus. The trajectory goes from this unstable focus to a quasi-periodic attractor found by numerical simulation and marked by diamonds, which represent the maximal and minimal amplitudes of the positive part of the time series envelope. The example time series and its Fast Fourier Transform (FFT) for $\Omega=2.75$ are both presented in Fig. 3. The same type of motion is obtained for the part of the curve from point $\mathrm{E}$ to $\mathrm{F}$. The quasi periodic motion goes to periodicity through the second kind of reverse Hopf bifurcation (a Neimark-Sacker bifurcation) at points B and E. At these points the selfexcitation frequency is quenched by the external force, and the beam vibrates periodically in the intervals $\mathrm{BC}$ and DE. In these regions the roots of the characteristic equation are conjugate complex numbers, or real numbers with negative real parts. Numerical simulations confirmed periodic motions in this area, marked by a circle in Fig. 2. Figure 4 presents the time series and FFT for a harmonic beam response for the selected frequency of excitation $\Omega=3.06309$. Solutions from points $\mathrm{C}$ to $\mathrm{D}$ are unstable with the roots of the characteristic equation being real numbers with different signs which correspond to an unstable saddle point. The effect of amplitude jumps occur, over a very narrow range of excitation frequency $\Delta \Omega=0.033$. If the frequency of the excitation is increased the beam amplitude jumps from $C$ to the upper branch next to $D$. If the frequency is decreased the amplitude jump is from point $\mathrm{D}$ to the lower branch next to point $\mathrm{B}$.

The beam model is strongly nonlinear. High and positive value of parameter $\gamma_{1}$ may suggest that the analytical curve should have the stiffening characteristic, inclined in the direction of higher frequencies. However, the results presented in Fig. 2 show a tendency opposite to this intuition. The curve has a softening characteristic which can be 


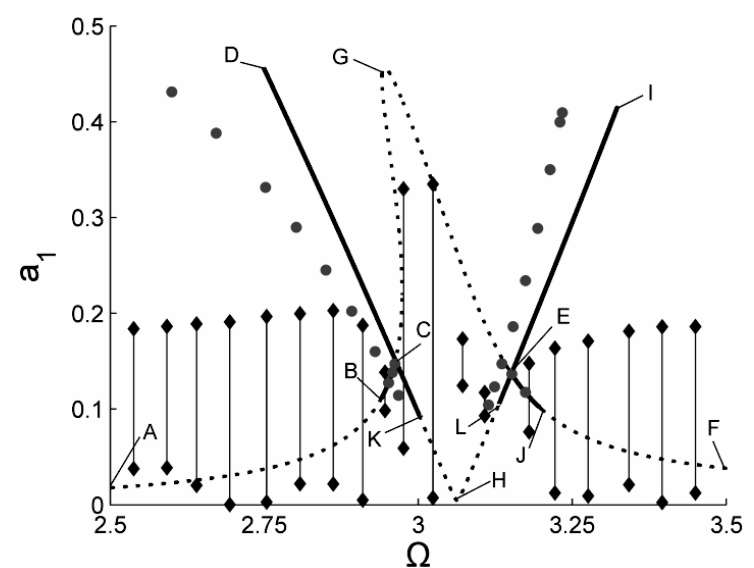

Fig. 5. Analytical and numerical results for the system with control. Analytical solution: solid line - stable solution, dotted line - unstable solution. Numerical results: circle markers - amplitude of periodic motion, diamond markers - maximum and minimum amplitude of positive part of envelope of quasi-periodic motion; $x_{0}=0.01, \alpha_{2}=$ 0.00306 .

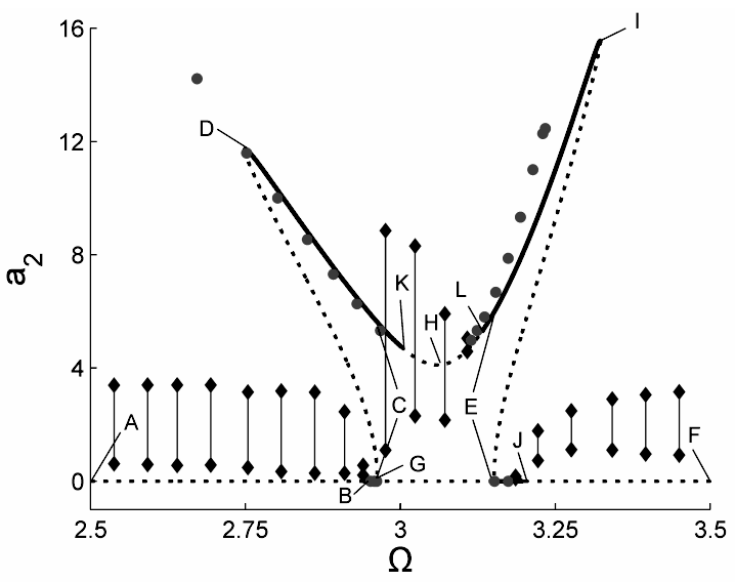

Fig. 6. Analytical and numerical results for the NSC controller response. Analytical solution: solid line - stable solution, dotted line unstable solution. Numerical results: circles - amplitude of periodic motion, diamonds - maximum and minimum amplitude of positive part of envelope of quasi-periodic motion; $x_{0}=0.01, \alpha_{2}=0.00306$.

explained by the strong influence of the nonlinear inertia terms. However, the performed numerical calculations for different structural parameters (beam's length and top mass values), show the final direction of the curve depends on the influence of both the geometrical (parameter $\gamma_{1}$ ) and the inertia (parameter $\delta$ ) nonlinearities.

\subsection{Nonlinear system with nonlinear saturation control}

In order to control both the self- and externally excited vibrations of the cantilever beam a nonlinear saturation controller is proposed. This method has been successfully applied to systems with positive viscous damping. In such a case provided that the parameters of the controller are properly chosen, the dynamic properties of the plant can be changed in the resonance region considered. Generally the literature provides recommendations for the case when the controlled object (the plant) is modelled by a linear system. If the desire is to minimise vibrations for a selected excitation frequency $\Omega$, then the controller should have a natural frequency of $\omega_{02}=\Omega / 2$ and the damping coefficient $\alpha_{2}$ should be close to zero (Pai et al. [14], Mitura et al. [9]). There are no literature studies for the NSC control of nonlinear plant with both external and self-excitations. In this section the effectiveness of saturation control of a strongly nonlinear beam with self and external excitations is tested. The parameters of the NSC controller are taken to minimise the beam response close to the first natural frequency $\omega_{01}=3.0631$. Therefore the natural frequency of the controller is tuned to $\omega_{02}=\omega_{01} / 2$ and its damping coefficient is assumed to have a small value of $\alpha_{2}=0.00306$. The analytical results and their numerical verification are presented in Fig. 5 for the beam and in Fig. 6 for the controller response. A solid line denotes stable analytical results and the dotted line depicts unstable analytical solutions, respectively. The solutions of the beam's response, the curve A-B-C-G-E-J$\mathrm{F}$ in Fig. 5, corresponds to the characteristics of the system without NSC control, as in Fig. 4, but in contrast to the system without control they are unstable in the interval C-G-E. In general, for the curve A-B-C-G-E-J-F the NS controller is not activated. The controller's amplitudes for this curve are equal to zero (Fig. 6), but it is worth mentioning that from point $\mathrm{B}$ to $\mathrm{C}$ and from $\mathrm{J}$ to $\mathrm{E}$ the zero controller solutions are stable (Fig. 6). Indeed, in this area the controller is deactivated, and its amplitude values obtained from direct numerical simulation (marked by circles) are equal to zero. The unstable solutions (A-B, J-F and C-G-E) go to quasi-periodic motion, both for the beam and the controller. The quasi-periodic response is marked by vertical lines with diamonds which define the maximal and minimal values of the amplitudes.

Taking as an example the controller's response for $\Omega=2.75$ it can be noticed that the spectrum does not have a frequency peak of $\omega_{02}=1.531545$ (Fig. 7). This is untypical behaviour of the NSC controller. This nonstandard effect leads to an increase in the beam vibrations. Comparing the amplitudes of the system with control (Fig. 5), and 


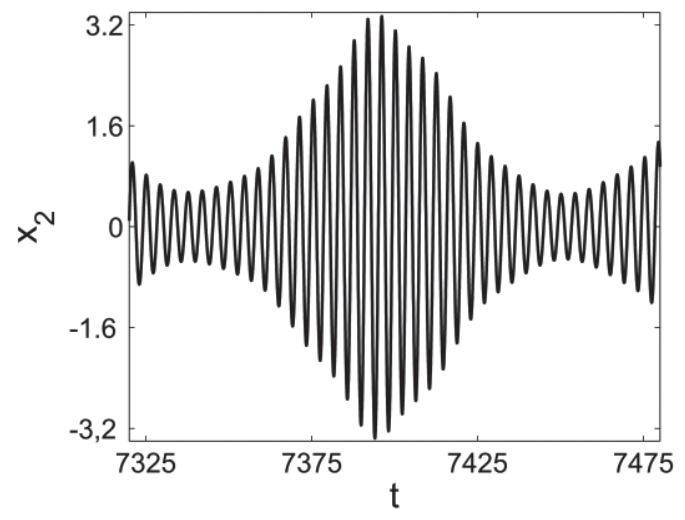

(a)

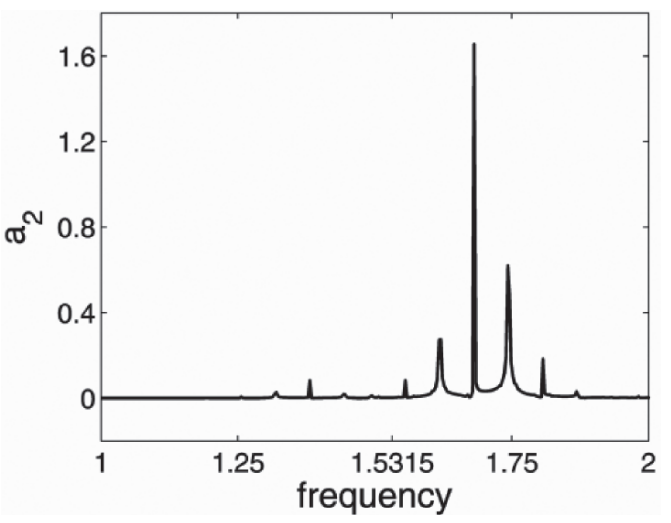

(b)

Fig. 7. Time series (a) and FFT (b) for quasi-periodic controller response; $x_{0}=0.01, \Omega=2.75$.

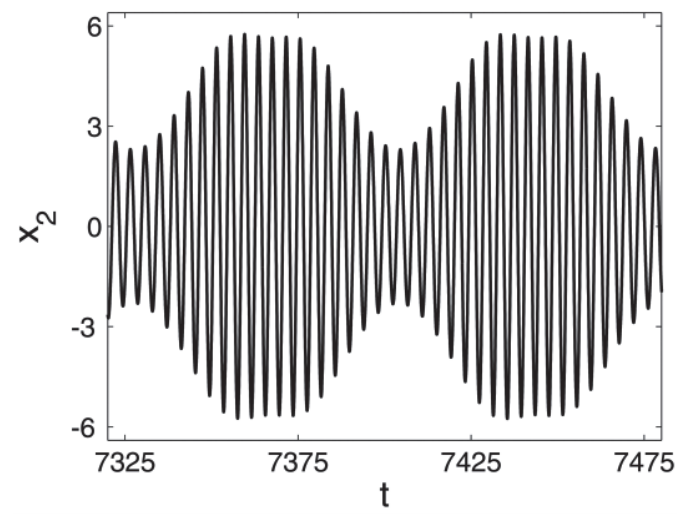

(a)

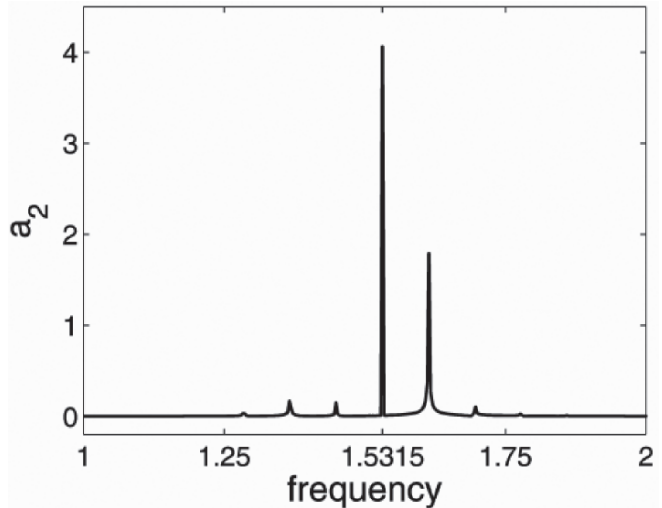

(b)

Fig. 8. Time series (a) and FFT (b) for quasi-periodic controller response; $x_{0}=0.01, \Omega=3.06309$.

the quasi-periodic motion of the system without control (Fig. 2), one sees a larger beating phenomenon, but with maximum amplitudes of the envelope at the same level.

The region where the controller is activated is shown by the curve D-C-K-H-L-E-I in Fig. 5. As can be seen the NSC controller makes the solutions located close to the natural frequency unstable (part $\mathrm{H}$ ), which results in an undesired large, quasi-periodic, beam response. The controller does not work properly in this case and its response degenerates to quasi-periodicity.

Analysing the spectrum of the quasi-periodic time series it can be observed that the peak at frequency $\omega_{02}$ always exists in that domain, as shown in Fig. 8 for $\Omega=3.06309$. In the ranges D-C and E-I there are two solutions, one stable and one unstable, but the amplitudes of both solutions have the same values. Therefore in Fig. 5 only the stable solutions are visible. The responses in the intervals C-D and E-I are not desirable because the beam response is larger there than for the system without control. Vibration reduction occurs for the frequency range in the domain C-K-H-L-E, however only the solutions in the C-K and E-J domains are actually stable. Around the point $\mathrm{H}$ (in the domain K-H-L) the beam's amplitudes are close to zero, but unfortunately they are unstable.

In order to change the unwanted situation the analytical and numerical calculations have been repeated for higher values of controller damping, $\alpha_{2}=0.106$. The results obtained present an improvement to the controller's performance. At first the solutions C-D and E-I from Fig. 5 are reduced (Fig. 9), and the arms of the curve are shorter. Secondly the region C-K-H-L-E in Fig. 5 is stable in this case. As regards the controller, we want it to be activated, so its trivial solution should not be stable. In fact the unwanted stable trivial solutions have also been eliminated as is visible in Fig. 10. One also notices from Fig. 9 that the beam's response in no larger than that of the system without 


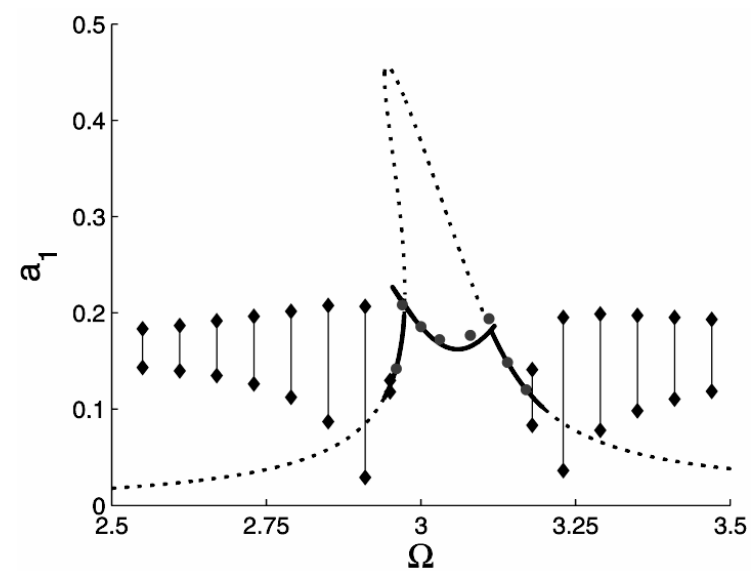

Fig. 9. Analytical and numerical results for the system with control. Analytical solution: solid line - stable solution, dotted line - unstable solution. Numerical results: circles - amplitude of periodic motion, diamonds - maximum and minimum amplitude of positive part of envelope of quasi-periodic motion; $x_{0}=0.01, \alpha_{2}=0.106$.

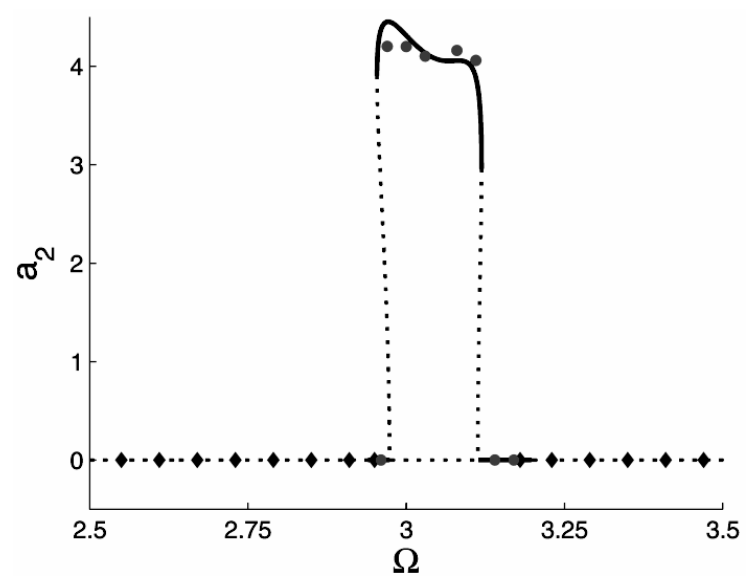

Fig. 10. Analytical and numerical results for the NSC controller. Analytical solution: solid line - stable solutions, dotted line - unstable solutions. Numerical results: circles - amplitude of periodic motion, diamonds - maximum and minimum amplitude of positive part of envelope of quasi-periodic motion; $x_{0}=0.01, \alpha_{2}=0.106$.

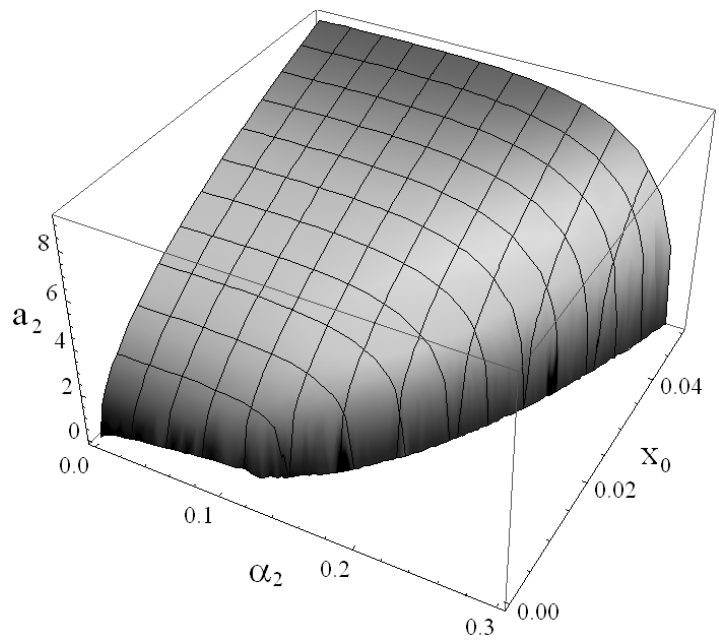

Fig. 11. Controller response versus controller damping and amplitude of excitation; $\Omega=3.06309$.

control, for the full frequency domain.

The approximate analytical results of Eq. (23) may be used to determined the influence of the controller damping $\alpha_{2}$ and the amplitude of excitation $x_{0}$ on the controller response. It is assumed that the structure is perfectly tuned, thus $\Omega=\omega_{01}=2 \omega_{02}$. After some simple calculations it is shown that the amplitude of the controller is a function of damping $\alpha_{2}$ and the amplitude of excitation $x_{0}$. The results are presented in Fig. 11. It is shown there that for small amplitudes of excitation and a big value of damping the controller is not activated. The second observation is that if the controller is activated its response for small damping values is almost independent of the $\alpha_{2}$ values, and, after a certain threshold of $\alpha_{2}$, the amplitude goes to zero. As regards the amplitude of excitation a significant influence from this is also observed.

It is possible to determine an analytical formula based on Eq. (23) for the critical value of the amplitude of excitation (i.e. the threshold) when the controller is activated, as follows

$$
x_{0}>\frac{\alpha_{2} \sqrt{16 g_{2}^{4} \alpha_{1}^{2}-24 g_{2}^{2} \alpha_{1} \alpha_{2}^{2} \beta_{1} \omega_{01}^{4}+\alpha_{2}^{4} \omega_{01}^{2}\left(9 \gamma_{1}^{2}-12 \gamma_{1} \delta \omega_{01}^{2}+4 \delta^{2} \omega_{01}^{4}+9 \beta_{1}^{2} \omega_{01}^{6}\right)}}{4 g_{2}^{3} \mu}
$$




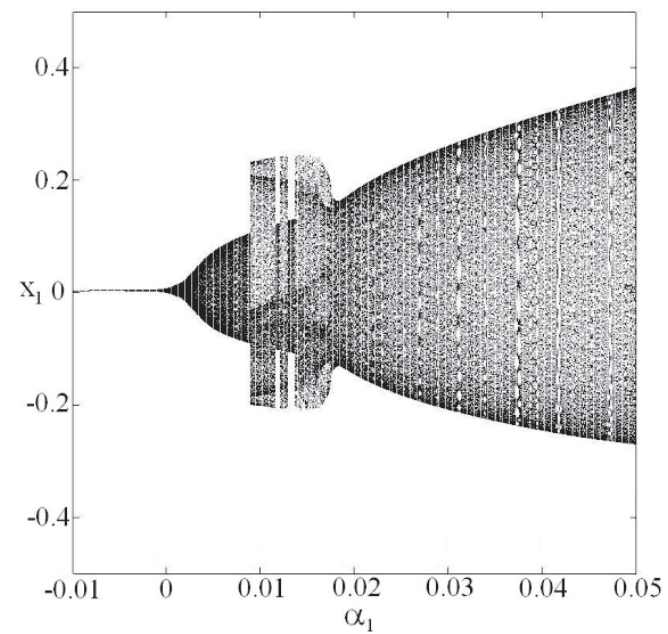

(a)

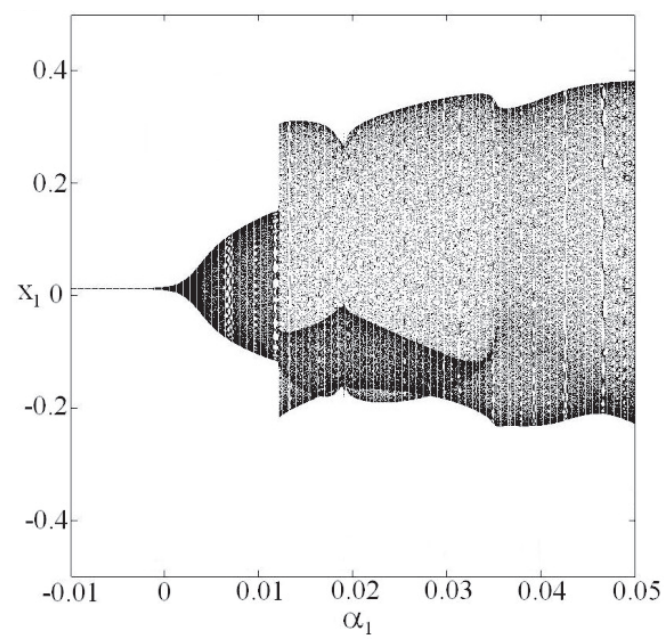

(b)

Fig. 12. Bifurcation diagrams of the beam response versus the self-excitation coefficient $\alpha_{1}$, (a) $x_{0}=0.01$, (b) $x_{0}=0.02$.

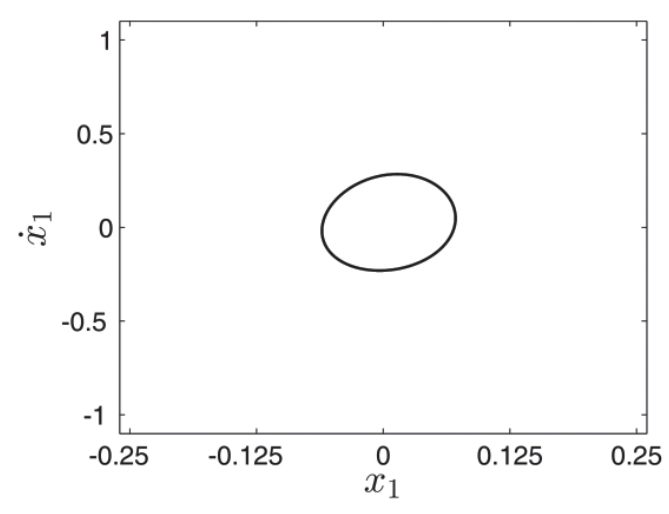

(a)

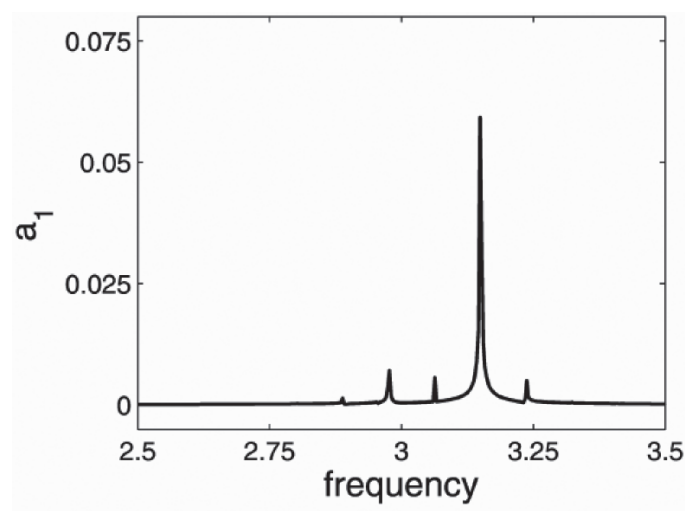

(b)

Fig. 13. Poincaré map (a) and fast Fourier transform (b) of the beam response; $x_{0}=0.01, \Omega=3.06309, \alpha_{2}=0.00306, \alpha_{1}=0.005$.

If the amplitude of excitation satisfies condition Eq. (26) then the controller is activated. When the amplitude of excitation is smaller than predicted by condition Eq. (26), the response of the controller is equal to zero. However, activation of the controller is the necessary but not sufficient condition to cause of the reduction of beam vibrations (Figs 5 and 6).

\section{Numerical bifurcation analysis}

In order to investigate influence of the most important structural parameters advanced numerical bifurcation analysis, using the 'Dynamics' software (Nusse, York [12]) has been performed. Figure 12 presents bifurcation diagrams obtained for the controller damping ratio $\alpha_{2}=0.00306$ when the structure is perfectly tuned $\left(\Omega=\omega_{01}=2 \omega_{02}\right)$. Two amplitudes of excitation are taken into account, $x_{0}=0.01$ (Fig. 12(a)) and $x_{0}=0.02$ (Fig. 12(b)). The stroboscopic projection against frequency $\Omega$ of the beam's response is plotted against the negative damping coefficient. When $\alpha_{1}<0$ the beam's damping is positive, and self-excitation doesn't occurs. In this case the NSC algorithm works properly and a low level of beam response is ensured.

For positive values of $\alpha_{1}$ a non-periodic response is observed, shown as a black area in Fig. 12. To recognise the type of system response the Poincaré maps are plotted for selected $\alpha_{1}$ values. Figure 13 presents the results obtained 


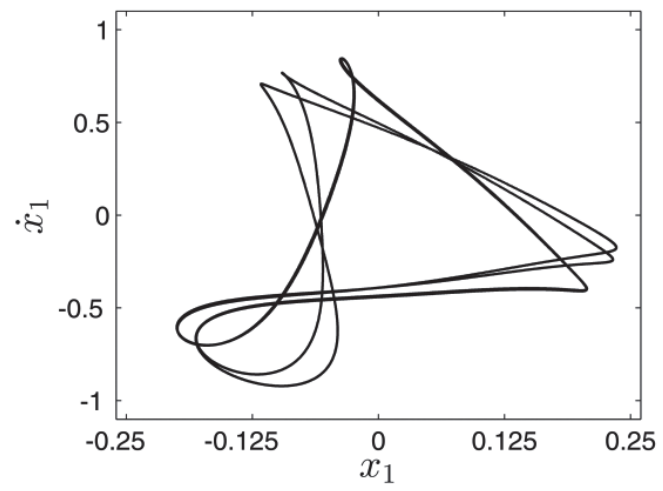

(a)

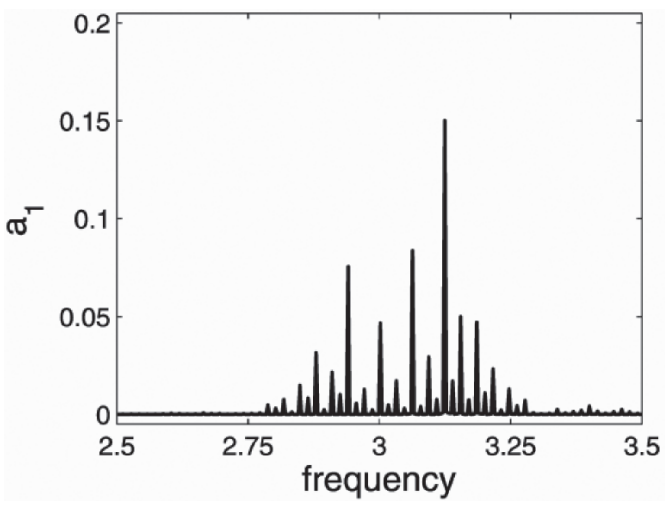

(b)

Fig. 14. Poincaré map (a) and fast Fourier transform (b) of the beam response; $x_{0}=0.01, \Omega=3.06309, \alpha_{2}=0.00306, \alpha_{1}=0.01$.

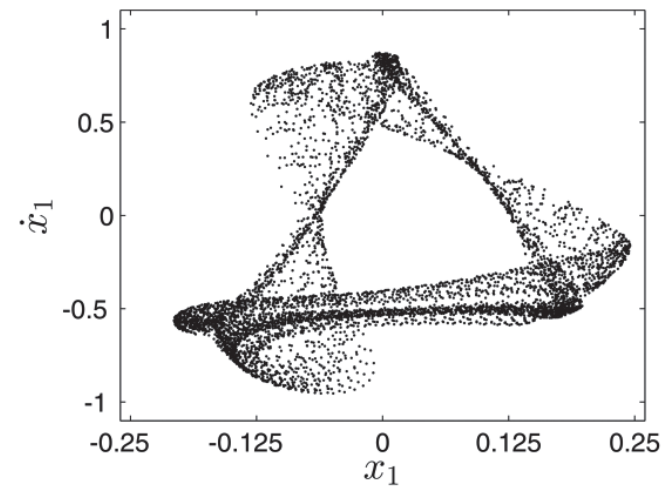

(a)

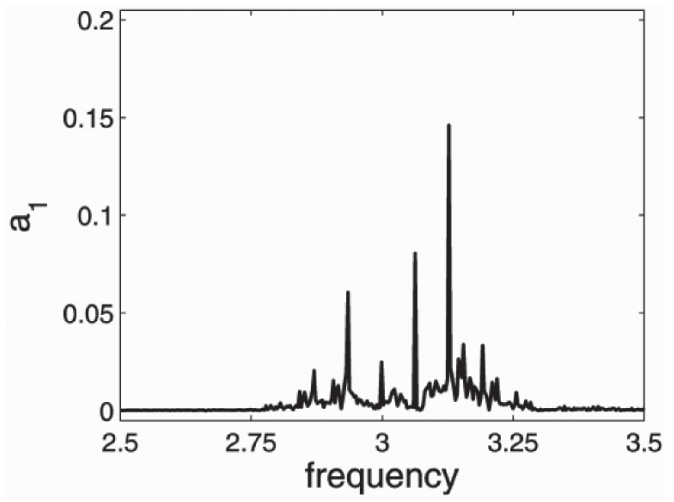

(b)

Fig. 15. Poincaré map (a) and fast Fourier transform (b) of the beam response; $x_{0}=0.01, \Omega=3.06309, \alpha_{2}=0.00306, \alpha_{1}=0.015$.

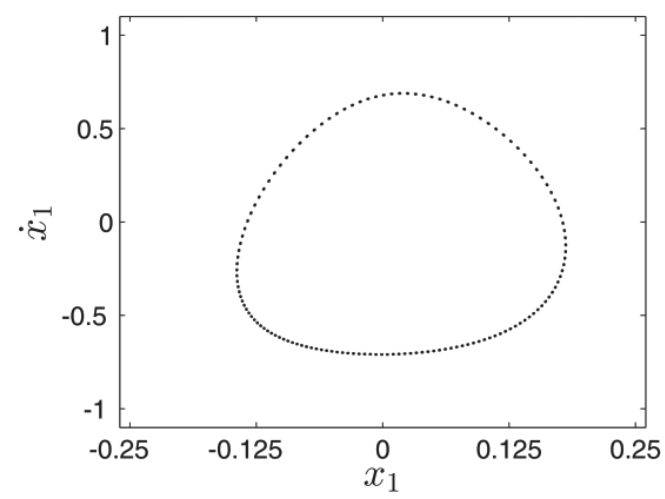

(a)

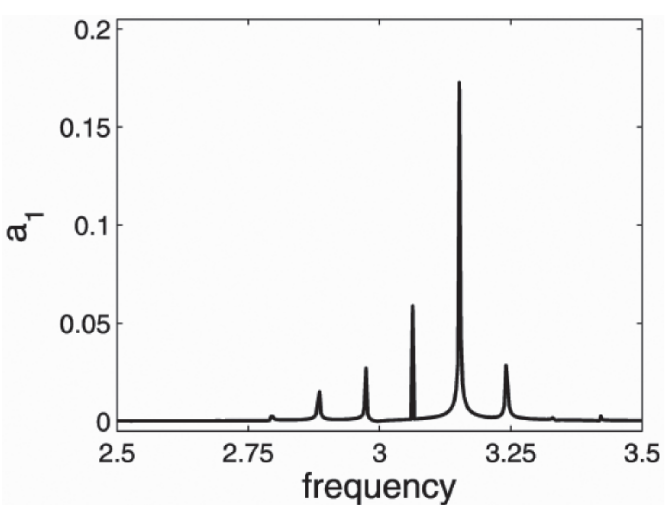

(b)

Fig. 16. Poincaré map (a) and fast Fourier transform (b) of the beam response; $x_{0}=0.01, \Omega=3.06309, \alpha_{2}=0.00306, \alpha_{1}=0.02$.

for the amplitude of excitation $x_{0}=0.01$ and negative damping for $\alpha_{1}=0.005$. It is clearly visible that the response of the beam is quasi-periodic. For $\alpha_{1}=0.01$ more complex dynamics are observed (Fig. 14) but the response is still quasi-periodic. FFT shows more peaks comparing with the situation presented in Fig. 13. The increase in parameter $\alpha_{1}$ to 0.015 leads to chaotic behaviour of the system (Fig. 15), for which the attractor on the Poincaré map has a 
fractal structure with a broad frequency spectrum. Further increase in the negative damping coefficient leads again to a quasi-periodic response (Fig. 16).

\section{Conclusions}

This paper deals with the dynamics and control of a nonlinear beam which is simultaneously self- and externally excited. It has been shown that interactions between self- and external excitation lead to instabilities in the neighbourhood of the fundamental resonance zone. After transitioning through a second kind of Hopf bifurcation the system may then vibrate quasi-periodically.

In order to reduce vibrations a nonlinear saturation controller is applied and its effectiveness is tested. The analytical and numerical solutions which have been obtained show that the controller may work ineffectively when operating close to the natural frequency region. In this region the steady state solutions are unstable and instead a quasi-periodic response is obtained. We may conclude that close to the resonance, and for small controller damping, the quasi-periodic response of the beam may occur, leading to a dangerous increase in beam vibrations. This is in contrast to the case of the structure with positive linear damping where minimization of the controller damping improves the NSC efficiency. To avoid this situation the influence of controller damping is studied in detail. The results show that the increase in controller damping may cancel the undesirable instability. Therefore to get use for vibration reduction the damping of the controller with respect to self- and external excitation should be properly selected to avoid system instabilities close to the fundamental natural frequency. Damping of the controller should be selected as small as possible but above the threshold depending on the intensity of self-excitation. If the frequency of the controller, which should be at half of the plant frequency, is not well tuned, or the external excitation is far from the resonance zone, then the beam's response is higher than for the structure without control. Bifurcation analysis also predicts chaotic dynamics of the system when self-excitation is varied over a wide range.

\section{Acknowledgments}

The first, third and fourth authors would like to acknowledge the financial support of Structural Funds in the Operational Programme - Innovative Economy (IE OP) financed from the European Regional Development Fund Project "Modern material technologies in aerospace industry", Nr POIG.01.01.02-00-015/08-00 is gratefully acknowledged.

\section{References}

[1] Abadi, Nonlinear Dynamics of Self-Excitation in Autoparametric Systems, University of Utrecht, Netherlands, 2003.

[2] M.P. Cartmell, An Introduction to Linear, Parametric and Nonlinear Vibrations, Chapman \& Hall, London, 1990.

[3] A.P. Duquette, K.L. Tuer and M.F. Golnaraghi, Vibration control of a flexible beam using a rotation internal resonance controller, part 1: Theoretical development and analysis, Journal of Sound and Vibration 167(1) (1993), 41-62.

[4] A.P. Duquette, K.L. Tuer and M.F. Golnaraghi, Vibration control of a flexible beam using a rotation internal resonance controller, part 2: Experiment, Journal of Sound and Vibration 167(1) (1993), 63-75.

[5] A.A. El-Badawy and T.N. Nasr El-Deen, Quadratic nonlinear control of a self-excited oscillator, Journal of Vibration and Control 13 (2007), 403-414.

[6] M.F. Golnaraghi, Regulation of flexible structures via nonlinear coupling, Dynamics and Control 1 (1991), 405-428.

[7] L. Jun, H. Hongxing and S. Rongying, Saturation-based active absorber for a non-linear plant to a principal external excitation, Mechanical Systems and Signal Processing 21 (2007), 1489-1498.

[8] L. Jun, L. Xiaobin and H. Hongxing, Active nonlinear saturation-based control for suppressing the free vibration of a self-excited plant, Communications in Nonlinear Science and Numerical Simulation 15 (2010), 1071-1079.

[9] A. Mitura, J. Warminski and M. Bochenski, Active vibration suppression by application of macro fiber composite, Machine Dynamics Research 35(2) (2011), 55-61.

[10] A. Nayfeh, Nonlinear Interactions, Analytical, Computational and Experimental Methods, Wiley, 2000.

[11] A. Nayfeh, Problems in Perturbations, John Wiley \& Sons, 1985.

[12] H.E. Nusse and J.A. York, Dynamics: Numerical Explorations, Springer, New York, 1994.

[13] S.S. Oueini, A.H. Nayfeh and J.R. Pratt, A nonlinear vibration absorber for flexible structures, Nonlinear Dynamics 15 (1998), $259-282$. 
[14] P.F. Pai, B. Wen, A.S. Naser and M.J. Schulz, Structural vibration control using PZT patches and non-linear phenomena, Journal of Sound and Vibration 215(2) (1998), 273-296.

[15] L. Pust and A. Tondl, System with a non-linear negative self-excitation, International Journal of Non-Linear Mechanics 43 (2008), $497-$ 503.

[16] K. Szabelski and J. Warminski, Vibrations of a non-linear self-excited system with two degrees of freedom under external and parametric excitation, International Journal of Nonlinear Dynamics 14 (1997), 23-36.

[17] K. Szabelski and J. Warminski, The self-excited system vibrations with the parametric and external excitations, Journal of Sound and Vibration 187(4) (1995), 595-607.

[18] K. Szabelski and J. Warminski, The parametric self excited non-linear system vibrations analysis with the inertial excitation, International Journal of Non-Linear Mechanics 30(2) (1995), 179-189.

[19] A. Tondl and H. Ecker, Cancelling of elf-excited vibrations by means of parametric excitation, in: Proceedings of the 1999 ASME Design Engineering Technical Conferences, Las Vegas, Nevada, USA, (12-15 September 1999).

[20] A. Tondl and R. Nabergoj, The effect of parametric excitation on a self-excited three-mass system, International Journal of Non-Linear Mechanics 39 (2004), 821-832.

[21] F. Verhurlst, Quenchening of self-excited vibrations, Journal of Engineering Mathematics 53 (2005), 349-358.

[22] J. Warminski, M. Bochenski, W. Jarzyna, P. Filipek and M. Augustyniak, Active suppression of nonlinear composite beam vibrations by selected control algorithms, Communications in Nonlinear Science and Numerical Simulation 16 (2011), 486-508.

[23] J. Warminski, Regular, chaotic and hyperchaotic vibrations of nonlinear systems with self, parametric and external excitations, Scientific Journal Facta Universitatis 3(14) (2003), 891-905.

[24] J. Warminski, Synchronisation effects and chaos in van der pol-mathieu oscillator, Journal of Theoretical and Applied Mechanics 4(39) (2001), 861-884.

[25] J. Xu, K.W. Chung and Y.Y. Zhao, Delayed saturation controller for vibration suppression in a stainless-steel beam, Nonlinear Dynamic $62(2010), 177-193$. 

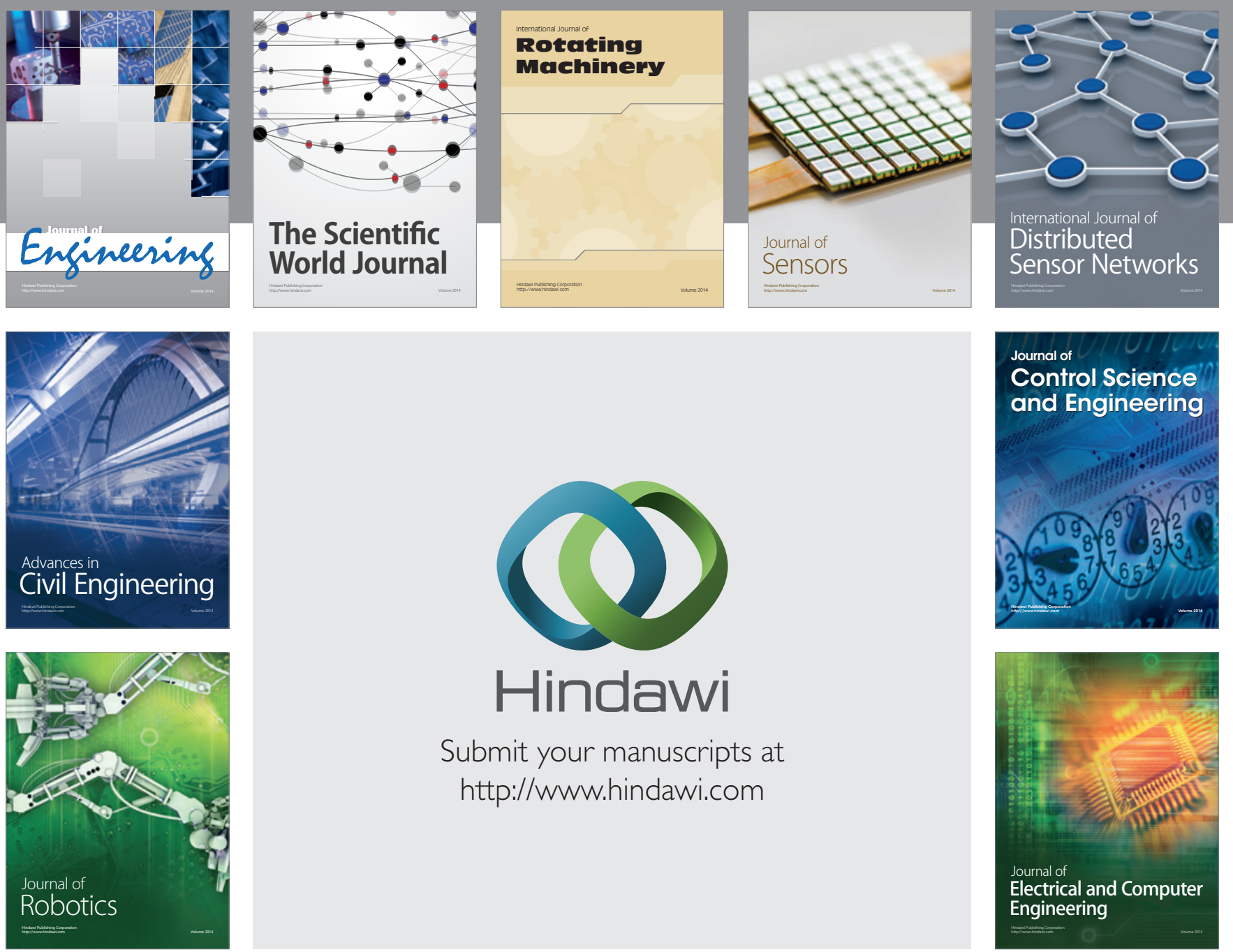

Submit your manuscripts at

http://www.hindawi.com
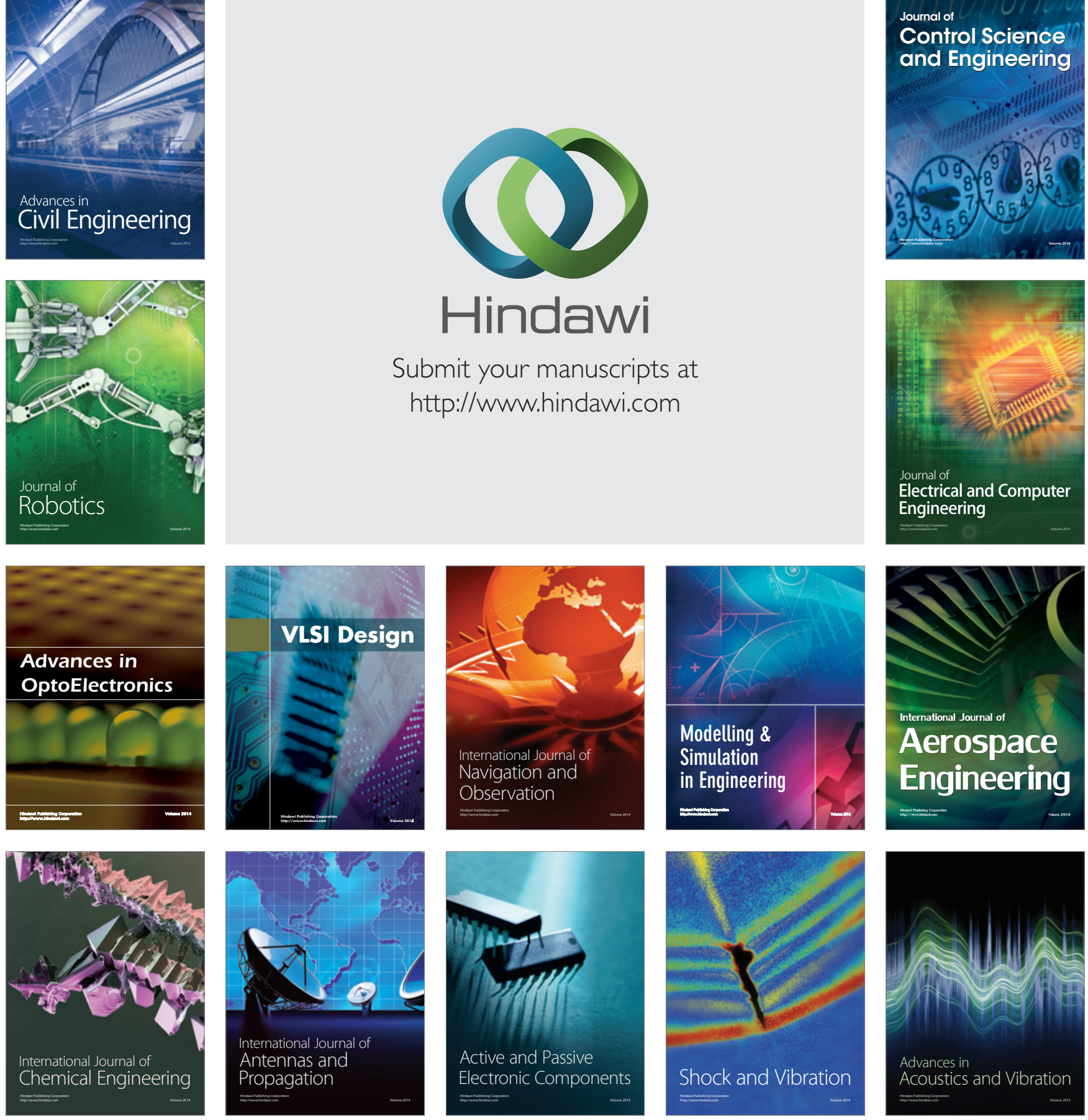